\section{Status epilepticus in the elderly: epidemiology, clinical aspects and treatment}

\author{
Telma M.R. de Assis,,1,2 Gersonita Costa, \\ Aroldo Bacellar,1 Marco Orsini,2 \\ Osvaldo J.M. Nascimento 2 \\ 1Department of Neurology, São Rafael \\ Hospital, Salvador, BA; 2 Federal \\ Fluminense University Pos-Graduating \\ Program on Neurology \Neuroscience, \\ Rio de Janeiro, Brazil
}

\section{Abstract}

The aim of the study was to review the epidemiology, clinical profile and discuss the etiology, prognosis and treatment options in patients aged 60 years or older presenting with status epilepticus. We performed a systematic review involving studies published from 1996 to 2010, in Medline/PubMed, Scientific Electronic Library on line (Scielo), LatinAmerican and Caribbean Center of Health Sciences Information (Lilacs) databases and textbooks. Related articles published before 1996, when relevant for discussing epilepsy in older people, were also included. Several population studies had shown an increased incidence of status epilepticus after the age of 60 years. Status epilepticus is a medical and neurological emergency that is associated with high morbidity and mortality, and is a major concern in the elderly compared to the general population. Prompt diagnosis and effective treatment of convulsive status epilepticus are crucial to avoid brain injury and reduce the fatality rate in this age group.

\section{Introduction}

The first descriptions of Status epilepticus (SE) were reported in antiquity but epilepsy and SE in the elderly have received little attention with few studies addressed to this specific population. ${ }^{1}$ Two large cooperative trials showed that up to $30 \%$ of elderly epileptic patients with recurrent partial seizures had a delayed diagnosis from the onset of epilepsy of more than one year.2,3 Up to $30 \%$ of acute seizures in the elderly present as SE. 4 Presentation of SE may be very subtle in this age-group, especially when it follows an acute injury of the central nervous system (CNS), such as stroke, anoxia, low anticonvulsant drug concentrations, and metabolic and alcohol-related insults. ${ }^{5,6}$ The aim of this review is to identify and discuss epidemiology, clinical features and treatment of SE in patients aged 60 years or older.

\section{Definition}

In 1981, the Commission on Classification and Terminology of the International League Against Epilepsy defined SE as a seizure that persists for a sufficient length of time or is repeated frequently enough that recovery between attacks does not occur. ${ }^{1,7}$ This definition was based on the persistence or repetition of the epileptic seizures rather than on the duration, although experts agreed that it should last at least $30 \mathrm{~min}$.

Recently some authors have analyzed videoelectroencephalography recordings and have demonstrated that the majority of self-limiting seizures last no longer than a few minutes. ${ }^{1}$ Therefore, Lowenstein and colleagues proposed a new operational definition that characterized generalized convulsive status epilepticus (GCSE) in adults and older children (age $>5$ years old) as 5 min or more of continuous seizures, or two or more discrete seizures between which there is incomplete recovery of consciousness. ${ }^{8,9}$ The 2001 ILAE Task Force on Classification and Terminology did not establish a precise time limit and defined status as seizure that shows no clinical signs of arresting after a duration encompassing the great majority of seizures of that type in most patients or recurrent seizures without interictal resumption of baseline central nervous system function.1,10 However, in spite of this, the most used definition of SE is more than 30 min of either continuous seizure activity or intermittent seizures without full recovery of consciousness. $., 6,8$

Refractory status epilepticus (RSE) is defined as SE that does not respond after initial therapy with a minimum number of two or three anticonvulsant drugs (ACD) or SE with seizures minimum duration of 1-2 $\mathrm{h}$ despite treatment. 11

There is no clear definition of non-convulsive SE (NCSE), and controversy remains and is dependent on the electroencephalography. 12 NCSE is characterized by some clinically evident alteration in mental status or behavior from baseline, without signs of convulsions, lasting at least $30 \mathrm{~min}$, with a pattern of seizure activity on the electroencephalogram that disappears with the treatment and recovery of consciousness. 6,13

\section{Epidemiology}

Changes in the population demography profile in developed and developing countries
Correspondence: Osvaldo J.M. Nascimento, Rua Siqueira Campos, 53/1204, Copacabana, CEP 22031-070, Rio de Janeiro, RJ, Brazil.

Tel. +55.2122 .354855 .

E-mail: osvaldo_nascimento@hotmail.com

Key words: case fatality, elderly, epidemiology, epilepsy, status epilepticus, treatment.

Conflict of interests: the authors report no conflict of interests.

Acknowledgments: we thank Dr. Robert J. DeLorenzo, Department of Neurology, Virginia Commonwealth University, for his permission to reproduce the Figure 2 published in Neurology 1996;46:1029-1035. We also thank Viguera Editores S.L. for their permission to reproduce the Table 1 published in Rev Neurol 2003;36 (7): 661-679.

Received for publication: 3 March 2012.

Revision received: 23 July 2012.

Accepted for publication: 30 July 2012.

This work is licensed under a Creative Commons Attribution NonCommercial 3.0 License (CC BYNC 3.0).

(C) Copyright T.M.R. de Assis et al., 2012

Licensee PAGEPress, Italy

Neurology International 2012; 4:e17

doi:10.4081/ni.2012.e17

have revealed the fastest growth in the older age group; therefore, SE is likely to become a common problem and an important public health issue. ${ }^{5}$

The elderly have a greater risk of cerebrovascular diseases, and degenerative and metabolic disorders that contribute to seizures occurrence. In elderly patients, SE is a common life-threatening condition because $30 \%$ of acute symptomatic seizures arise as SE compared with $2-16 \%$ in epileptic patients. ${ }^{4,12}$

The prospective population study conducted in Rochester, Minnesota, USA, showed an annual SE incidence rate of 18.3 per 100,000 habitants, with a bimodal distribution curve (U), with the highest incidence rates seen in children under the age of one year and in adults aged 60 years and above. SE also lasted longer (at least $2 \mathrm{~h}$ ) in those populations. In this study, the cumulative incidence for 75 year olds was 4 per $1000.4,14$

The annual incidence rate of SE, in the epidemiological study of Richmond, Virginia, USA, was 86 per 100,000 in the 60 years and older age group, almost twice that of the general population, while the highest incidence was in the 70 years and older age group.6,15 The highest incidence rate of SE (156 per 100,000 ) was found in children between one and 12 months of age; nevertheless, when the data were analyzed according to age groups, 
with the pediatric group consisting of children aged from one month up to 16 years, the elderly age group (60 years and above) had the highest incidence rate (Figure 1) ${ }^{6}$

A study of GCSE conducted in California, found the highest incidence rate of GCSE among children under the age of 5 years $(7.5 / 100,000)$ and among the elderly aged 75 years and older $(22.3 / 100,000) .16$ The reason for the lower incidence of SE seen in this study may be partially explained by its retrospective nature and by the fact that only GCSE cases were included. ${ }^{16}$ A prospective study conducted in Italy showed incidence rate of 26.2 per 100,000 in patients aged over 60 years and 5.2 per 100,000 among younger age groups. ${ }^{6,17}$ In Germany, a prospective population study, revealed similar results to previous studies in the United States, with the highest incidence in the over 60 years age and group of 54.5 per 100,000 versus 4.2 per 100,000 in younger adults (18-59 years old). ${ }^{18}$ The EPISTAR cohort study showed the highest incidence rate of SE in children under one year age and a progressive increase after the age of 60 years. ${ }^{19}$

\section{Classification}

Until the 1960s, the term status epilepticus presupposed only the occurrence of GCSE although NCSE had already been described. Experts then went on to recognize several forms of SE and agreed that there are as many types of status as there are types of seizures. ${ }^{1}$ In general, SE can be classified according to clinical spectrum, type of seizure (convulsive $v s$ non convulsive) $)^{1}$ or on the basis of electrographic features (partial $v s$ generalized).$^{8}$ All forms of partial SE are classified clinically as non-convulsive. ${ }^{8}$

Although it has been proposed to classify SE according to several features, such as patient age, maturity of the central nervous system, electroclinical semiology, genetic factors and pathophysiology, for practical purposes the current diagnostic scheme that subdivides SE into convulsive and non-convulsive is more useful (Table 1). ${ }^{20}$ This limited classification does not consider the actual complexity and semiological variability of SE.1

While generalized SE, consisting of tonic and/or clonic motor activity, is the most common form of SE, in the elderly, partial SE with secondary generalization is the most common presentation (45\%), followed by partial (29\%) and generalized tonic-clonic. ${ }^{21}$

Elderly patients in SE often present with no convulsive activity or less apparent clinical manifestations that can be underecognized. The NCSE has been described as a relatively benign condition with no neuronal damage, because there are no adverse systemic consequences on its development such as occur in
CGSE, such as hyperthermia, acidosis, hyperkalemia, pulmonary or cardiovascular collapse. ${ }^{22}$ However, some authors found this condition a diagnostic challenge due to the absence of typical clinical seizure signs, its association with neuronal damage from abnormal persistent electrical activity, and for its interaction with acute neurological disorders, such as cerebrovascular accidents (CVAs) that may precipitate the SE. ${ }^{23}$ The complex partial status epilepticus (CPSE), one of the two major types of NCSE, is associated with serious morbidity and mortality, mainly in the geriatric population whose brain parenchyma is more sensitive to prolonged abnormal electrical activity, with harmful consequences to usual impaired baseline mental status leading to risk of permanent neurological damage. ${ }^{24}$

One observational prospective study determined the prevalence, clinical features and

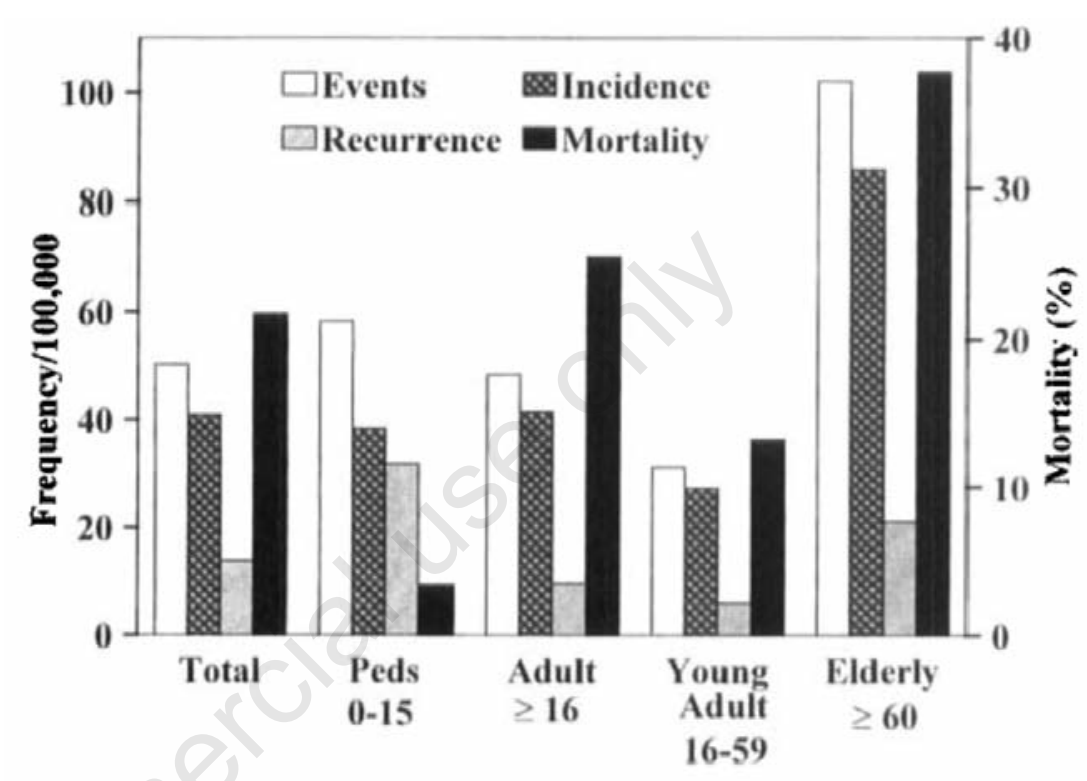

Figure 1. Frequency of status epilepticus (SE) events, incidence, SE recurrence, frequency, and SE mortality in Richmond for the total, pediatric (Peds), adult, young adult, and elderly populations. The data for events, incidence, and recurrence present the frequency per 100,000 per year. Mortality data express the percent mortality for each age group. Data from DeLorenzo et al.,1996.15 Reproduced with permission.

Table 1. Status epilepticus classification.

\begin{tabular}{ll}
\hline Convulsive SE & \\
Generalized & Tonic-clonic (primary, secondary) \\
& Subtle (oligosymptomatic) \\
& Myoclonic \\
& Tonic \\
Simple partial & Clonic \\
& Somatomotor \\
& Postural motor \\
& Epilepsia partialis continua
\end{tabular}

\begin{tabular}{ll} 
Non-convulsive SE & Absences (typical/atypical) \\
Generalized & Somatosensory \\
Simple partial & Sensory \\
& Autonomic (vegetative) \\
& Psychic (affective) \\
& Dysphasic (aphasic) \\
& Motor inhibitory \\
& Combination of all above \\
Complex Partial & \\
\hline SE, status epilepticus. Adapted from: Tejeiro J, Gómez-Sereno B, Status epilepticus. Rev Neurol 2003;30:661-79. Permission obtained from
\end{tabular}
Viguera Editores S.L. 
course of NCSE in older inpatients with acute unexplained change in mental, cognitive or behavioral status and diagnosed 7 patients with NCSE. All patients presented with acute altered mental state: confusion, stupor or coma, without clinical convulsive activity. One of them had a previous history of epilepsy. In 3 patients, the presenting symptom was refusal to eat. The other patients had NCSE during the course of several medical conditions, such as acute and chronic renal insufficiency, dementia and sepsis. Time until diagnosis was 1-5 days (mean 3 days). All patients had generalized epileptiform activity on electroencephalogram (EEG) that disappeared with intravenous diazepam injection. The authors highlighted the difficulties of diagnosis and the important role of the EEG in the evaluation of acute mental and behavior changes in the geriatric population. ${ }^{24}$ In a study analyzing the causes of diagnostic and treatment delay in NCSE in the emergency room, Kaplan has identified delays from days to weeks caused by assigning the confusional status to a metabolic encephalopathy, a post-critical condition, a psychiatric cause, an intoxication, a mutism state or to an aphasia. ${ }^{25,26}$ In another case series study of 22 elderly outpatients with acute altered mental state, the authors demonstrated that the ictal basis underlying confusion was not recognized for up to five days. All the patients had protracted confusion and the semiology was impaired attention and concentration, reduced speech to simple semiautomatic phrases or gestures. Subtle ictal manifestations were light gaze preference, low-amplitude fragmentary myoclonic jerks, typically in the face, eyelids or hands, and at times associated with hand automatism. Some patients had contralateral apraxia and those who were able to respond to commands had impersistence of motor activity. All patients had electrographic seizures and were treated successfully with antiepileptic drugs. After discharge, 14 patients had a reduction in cognitive abilities or a worsening of previously identified minimal cognitive impairment. The authors reported the important role of the EEG and video-EEG in quickly identifying the ictal nature of the protracted confusion. 27

The Intensive Care Unit (ICU) is a common setting for the occurrence of NCSE in the elderly. These patients are often comatose and may have severe metabolic, neurological or systemic disease that predispose to SE. 5 NCSE was discovered in $8 \%$ of comatose ICU patients. ${ }^{12}$

\section{Etiologies}

Etiologies of SE are age-dependent and determine the prognosis. ${ }^{28}$ In the elderly, the most important causes of SE are stroke, hypoxia, metabolic insults and low anticonvulsant drug concentrations. ${ }^{2}$ In a retrospective study of 102 patients with SE who had their first seizure after 60 years of age, cerebrovascular disease was responsible for $35 \%$ of seizures, followed by head trauma (21\%). ${ }^{29}$ The study conducted in Richmond, Virginia, USA, showed that in patients over 60 years of age, $35 \%$ of the SE were caused by acute CVA and $26 \%$ by remote $\mathrm{CVA},{ }^{30}$ with a total of $61 \%$ of SE secondary to stroke. Others etiologies included hypoxia (17\%), metabolic disorders (14\%), alcohol-related (11\%), tumor (10\%), infection (6\%), anoxia (6\%), hemorrhage (5\%), CNS infection (5\%), trauma (1\%), idiopathic (1\%) and other (1\%). The population study of Rochester, Minnesota, USA, added dementia to the list of SE etiology. ${ }^{14}$ One population study completed in California identified stroke as a common cause of generalized status epilepticus (GSE) in older patients. ${ }^{16}$ In the 2000 EPISTAR study, most of the cases of SE in the elderly occurred in close association with an acute cerebral insult such as CVA, CNS infection, alcohol-related and metabolic insults and drug withdrawal. 19

One large, prospective, hospital-based stroke cohort showed that SE was common among patients with poststroke seizures and, although the immediate prognosis was poor, SE as the presenting sign did not necessarily predict subsequent epilepsy, though if seizures recurred, they were severe. 31

In the consecutive series of 22 patients with acute protracted ictal confusion, the causes to the event were: remote traumatic brain injury in 3 patients, Alzheimer-type senile dementia in 4 patients, lupus erythematosus and lung cancer in one patient each, and primary generalized epilepsy in 2 patients. ${ }^{27}$ Another study evaluated 5 elderly patients with confusional state in the context of presumed dementia who were later found to have complex partial seizures with pseudodementia. ${ }^{32}$

The incidence of SE after stroke is low or is not cited at all. 31,33 It occurs in only $1.5 \%$ of the overall stroke population but in more than $10 \%$ of patients with stroke-related seizures. ${ }^{34}$ Association of SE with symptomatic seizures due to CVA has an overall incidence rate at 14$27 \%$. The heterogeneity of the reported percentages is probably related to the differences in study designs. ${ }^{31}$ Prognosis of SE after stroke is poor with a high mortality rate and occurs more frequently at stroke onset. 33,34

\section{Mortality}

Seizure duration, clinical presentation, age and specific etiology are strong predictors of mortality in SE. 6
Data from the studies of Richmond, Virginia, USA, corroborated the results of previous studies that demonstrated older age groups have the highest mortality rates related to SE. 15 In the elderly population, SE was associated with a mortality at $38 \%$, approaching $50 \%$ in patients over 80 years of age. ${ }^{20,27}$ In the elderly, while the GCSE is associated with a high mortality rate of $49 \%$, the partial status epilepticus (PSE) has a mortality rate of $30 \% .4$ The highest mortality rate is associated with SE secondary to anoxia (70\%), followed by infection and metabolic disorders (40-50\%). ${ }^{35}$ Towne et al. found $100 \%$ mortality rate in patients with anoxia. ${ }^{5,36}$ SE secondary to alcohol withdrawal or low levels of ACD has a mortality rate of less than $9 \%$. Symptomatic cases, the majority of which consist of prior CVA, have a mortality rate of $14 \% .^{6}$ One study conducted to ascertain whether the high mortality rate seen in patients with stroke and SE differs significantly from the mortality for stroke alone, demonstrated a nearly 3 -fold statistically significant increase in mortality in patients with SE associated with acute ischemic stroke $(\mathrm{P}<0.001)$. These findings would indicate a synergistic effect of $\mathrm{SE}$ in ischemic brain injury. ${ }^{37}$ The manifestations of SE in the elderly can be very subtle, particularly following a CNS insult such as transient anoxia. The clinical features may subside entirely after diffuse brain injury (anoxia) or after status has persisted for an extended period of time. The epileptogenic discharges may still be present on the EEG. The high mortality rate seen in these cases may be secondary to the primary anoxic injury or from a delay in recognizing status and initiating treatment. ${ }^{2}$

In the ICU, NCSE in the elderly has a mortality rate that approaches $36 \%$ as a result of the cause of the confusional state from the more severe underlying process and is not associated to SE duration.21 NCSE in the elderly is associated with a high rate of hospitalacquired infections, which may be fatal. In a study of 25 critically ill older patients presenting with NCSE, a mortality rate of $52 \%$ was found, and death was associated with the number of acute life-threatening medical problems. ${ }^{38}$ In this group of patients, treatment of NCSE with benzodiazepines increased the risk of death, and aggressive anticonvulsant therapy did not improve the outcome. 6,38

\section{Electroencephalogram}

Benign EEG variants with epileptiform morphology occur in all age groups. In the geriatric population, in addition to changes in the normal spectrum of EEG, there are three EEG variants (subclinical rhythmic electrical discharges of adulthood, wicket spikes, and small 
sharp spikes) that occur with greater frequency in older people and can potentially be misinterpreted as epileptiform abnormalities. ${ }^{39}$ In this age group, interictal epileptiform activity occurs less frequently than in younger age groups. A randomized, double-blind, parallel trial (VA Cooperative Study 428 Group) conducted to compare tolerability and efficacy of three monotheraphy treatments in elderly patients, found no epileptiform activity on routine EEGs in $25.3 \%$ of patients diagnosed with generalized tonic clonic seizures alone.40 Video-EEG monitoring and prolonged EEG recordings significantly increase the possibility of establishing a definitive diagnosis. ${ }^{41}$ In a study of 38 patients with NCSE conducted by Lowenstein and Aminoff, clinical presentation was subtle motor activity and depressed consciousness, and EEG was necessary for diagnosis. ${ }^{42}$ Lee studied 11 outpatients presenting with an acute onset of a prolonged confusional state, varying from mild disorientation to confusion and, some of them demonstrated atypical behavior, laughing, singing or inappropriately dancing. The author describes $1-2.5 \mathrm{~Hz}$ generalized spike-wave or multiple spike-wave discharges on EEG. The patients were successfully treated with intravenously administered diazepam followed by orally administered phenytoin sodium and phenobarbital. ${ }^{43}$ In the Richmond, Virginia, USA, study, 14\% of patients with clinical seizures ceased, but they were comatose, still had electrographic SE and $34 \%$ had recurrent seizures of which more than two-thirds were non-convulsive. 5

In a prospective study of 236 ICU patients in coma and with no overt clinical seizure activity, of which $38 \%$ were elderly, EEG monitoring was performed in all as part of coma evaluation. NCSE was diagnosed in $8 \%$ of all comatose patients. The EEG criteria for NCSE consisted of discrete electrographic seizures, continuous spike and wave activity, or rhythmic recurrent epileptiform activity. The authors showed the utility of routine EEGs in the evaluation of comatose patients even if clinical seizure activity is not apparent. ${ }^{44} \mathrm{~A}$ significant number of elderly patients in whom GCSE appears to have stopped, with apparent motor activity control, convert to electrographic SE. 5

\section{Treatment}

There is no established protocol for SE management in elderly patients. ${ }^{6}$ The Epilepsy Foundation of America and other institutions have suggested protocols for the treatment of SE that have been widely accepted or adapted. ${ }^{5}$ Although the objective of the treatment is expeditious cessation of clinical and electrical seizure, the therapeutic approach in the elder- ly is worthy of special attention. Biological issues, such as altered volume of distribution, lower protein binding, decreased renal elimination, decreased hepatic metabolism, decreased enzyme inducibility, and increased use of polypharmacy in this age group result in more complex pharmacokinetics in the elderly than in younger patients. 6

Despite defining SE as a 30 min duration, treatment should be initiated promptly for any convulsive seizure that has lasted at least ten minutes, or for repetitive seizures.5,6 Initially and without delay, general action should be taken: establish an airway, monitor oxygenation and vital signs, obtain an intravenous access, measure blood glucose levels, and take blood samples to measure blood count, serum electrolytes, and ACD levels. ${ }^{5,6}$

\section{Initial treatment (first-line)}

\section{Benzodiazepines}

Benzodiazepines have been used as firstline agents for aborting SE because they are fast acting and effective for all seizures types. ${ }^{45}$ Lorazepam has a longer duration of effect (12-24 h) than diazepam (15-30 min). Lowenstein demonstrated that when administered by paramedics to adults for out-of-hospital SE, lorazepam is more effective than diazepam at terminating $\mathrm{SE},{ }^{5}$ although it may cause a prolonged period of depressed consciousness. Diazepam is more lipid soluble and it rapidly enters the brain; however, it is rapidly redistributed to other fatty tissues which causes a fall in serum concentrations. Benzodiazepines tend to accumulate leading to respiratory suppression, hypotension and sedation. ${ }^{8}$ The brief therapeutic effect of diazepam may lead to recurrence of SE following a single initial dose. It is usually recommended that a longer acting non-lipophilic drug that does not promote accumulation after repeated use, such as phenytoin or fosphenytoin, be administered immediately after diazepam when seizures persist or even when they have been aborted.9,46

\section{Phenytoin and fosphenytoin}

Phenytoin has been shown to be effective in terminating GCSE in $41-90 \%$ of patients in some uncontrolled retrospective studies. ${ }^{8}$ The recommended loading dose of phenytoin is 20 $\mathrm{mg} / \mathrm{kg}$ intravenously (i.v.) in bolus in adults, and $15 \mathrm{mg} / \mathrm{kg}$ in the elderly, ${ }^{3}$ preferably without dilution, with a maximal rate of administration of $50 \mathrm{mg} / \mathrm{min}$ in adults and $20 \mathrm{mg} / \mathrm{min}$ in the elderly due to the possibility of occurrence of significant hypotension and arrhythmias.5,46 Fosphenytoin, the phosphate ester of phenytoin, unlike phenytoin, is a highly water-solu- ble prodrug, with a near physiological pH. After i.v. administration, fosphenytoin is rapidly and completely converted to phenytoin in the liver and other tissues in 8 min, 25 achieving therapeutic concentrations within $10 \mathrm{~min}$ when either fosphenytoin or phenytoin is administrated at maximal infusion rates.5,8 Approximately $1.5 \mathrm{mg}$ of fosphenytoin is equivalent to $1.0 \mathrm{mg}$ of phenytoin, and the dosage is expressed as the amount of phenytoin delivered as phenytoin equivalents (PE).5,6,8

Fosphenytoin can be infused at rates of up to $150 \mathrm{mg}$ PE per minute. Unlike phenytoin, it is not formulated with propylene glycol, allowing a faster infusion rate, better tolerability with fewer local side effects, and less toxicity from the drug vehicle.5,25 Fosphenytoin can be given intramuscularly, unlike phenytoin, with therapeutic levels reached in approximately 20-30 min. ${ }^{25,47}$ However, no clinically significant differences in adverse effects of hypotension or cardiac arrhythmias, as with phenytoin, were found, so the infusion rates should be slowed down if these conditions or prolongation of QT interval on the electrocardiogram (ECG) occur. ${ }^{8}$ In the elderly it is important to monitor ECG and blood pressure. ${ }^{5}$

\section{Phenobarbital}

In the elderly, phenobarbital can be given at a loading dose of $20 \mathrm{mg} / \mathrm{kg}$ and administered at a rate of $50-75 \mathrm{mg} / \mathrm{min}^{3}$ Sedation, apnea, along with hypotension, can occur with phenobarbital infusion, particularly if benzodiazepines were administered previously. 3,8 Therefore, especially in the elderly, intravenous phenobarbital loading requires careful monitoring for cardiac rhythm and of blood pressure, and the patient is usually intubated for airway support. 8

In a large 5-year, double-blind, multicenter trial, Veterans Affairs, 48 patients with GCSE were randomly assigned to receive initial treatment with one of four intravenous regimens: lorazepam, phenobarbital, phenytoin, or diazepam followed by phenytoin. In this study, many patients were elderly: median age 58.6 years in the overt convulsive SE group and 62.0 years in the subtle convulsive SE group. The success rate for initial treatment ranged from $64.9 \%$ to lorazepam, $43.6 \%$ to phenytoin, $55.8 \%$ to diazepam followed by phenytoin, and $58.2 \%$ to phenobarbital. When lorazepam and phenytoin were compared, lorazepam was significantly more effective $(\mathrm{P}=0.002)$, as initial i.v. treatment for overt GCSE. In the group of NCSE, no significant differences $(\mathrm{P}=0.91)$ were observed among the treatment groups and the success rate was only $14.9 \%$. There were no differences between the four treatments with respect to recurrence during the 12-h study period, the incidence of adverse reactions such as respiratory depression, cardiac arrhythmias or hypotension requiring treatment, or the outcome at 30 days. ${ }^{48}$ 


\section{New agents}

\section{Valproic acid}

Valproic acid (VA) is probably as effective as phenytoin and would be indicated in those patients who did not respond to first-line therapy or when these agents are contraindicated. ${ }^{46}$ A European study of 23 cases of SE which included patients with absence, tonic-clonic, myoclonic and partial SE, found that $82.6 \%$ of the patients responded within 20 min to a 15 $\mathrm{mg} / \mathrm{kg}$ i.v. valproic acid initial injection, followed $30 \mathrm{~min}$ after the bolus by an infusion of $1 \mathrm{mg} / \mathrm{kg} / \mathrm{h}$ for 5-6 h.5,6 Loading doses of i.v. valproic acid, as high as $60-70 \mathrm{mg} / \mathrm{kg}$, followed by maintenance infusion have also been demonstrated to be efficacious and safe in the treatment of SE.46,49 Because of rapid changes of total (unbound plus bound) and unbound valproic acid concentrations during rapid infusion, unbound valproic acid concentrations should be monitored when i.v. valproic acid is given to treat patient with SE. The low risk of hypotension, respiratory depression and sedation makes i.v. valproic acid a potential choice of treatment of SE in the elderly. ${ }^{5}$ In these patients, smaller doses of VA may be required to achieve a given serum concentration.5,6 The potential presence of inhibiting co-medications needs to be considered.6,49,50 Randomized studies are needed before the role of VA in overall SE treatment is established.6,46,49

\section{Levetiracetam}

Levetiracetam (LEV), chemically unrelated to established ACD, has favorable pharmacokinetic profiles and fewer interactions, being well tolerated in critically ill older patients. ${ }^{51}$ It has a wide range of action and can be effective for treatment of focal and generalized seizures. 51 The i.v. formulation is bioequivalent to oral tablets. Rosseti and Bromfield analyzed 13 episodes of SE in adult patients who were treated with oral LEV (mostly via nasogastric tube) in doses that ranged from 1000 to $6000 \mathrm{mg}$. Only 3 (23\%) were regarded as responders and in 4 patients (31\%) SE continued despite the administration of LEV. These patients had refractory SE requiring general anesthesia and ventilation. The authors concluded that LEV may be a useful alternative in SE treatment.6,52 In a retrospective observational study of 14 older patients (age range 6197 years), complete seizure control was achieved with relatively low doses of LEV (mean $1.643 \mathrm{mg} \pm 949.3 \mathrm{mg} /$ day) in $78.6 \%$ of patients and no serious side effects, except somnolence, were observed.53 One study reports the occurrence of NCSE in 2 patients treated with LEV $2000 \mathrm{mg} .{ }^{54} \mathrm{LEV}$ can be effective in the treatment of SE, and seems to be a reasonable practical alternative to multimorbid older patients who need i.v. treatment with an AED, although clinical experience is still very limited, especially in this patient population.

\section{Treatment of refractory status epilepticus}

Few studies have been conducted to evaluate the RSE as a serious clinical condition, despite an estimated frequency of RSE in patients with SE of 9-40\%.55 RSE is associated with a delay in beginning treatment and to nostructural causes of SE, such as hypoxicischemic encephalopathy, metabolic encephalopathy and SNC infection. 55

In an analysis of results of the Veterans Affairs Cooperative Study, the authors demonstrated that, when the initial treatment of SE fails, little is gained by using additional standard drugs. ${ }^{56}$ The study, conducted using the collected data from Veterans Affairs Cooperative Study to determine the efficacy of protocols to sequential treatment of $\mathrm{SE}$, showed that only $7 \%$ of overt convulsive SE and $3 \%$ of subtle SE responded to treatment with a second drug. 6,56 In the same study, it was also observed that the overall success of first drug treatment to overt SE was $55 \%$ and to subtle SE $14.9 \% .5$

General anesthetic agents such as pentobarbital, midazolam or propofol are recommended as therapeutic options to patients who continue to have seizures after the initial therapy with benzodiazepines and phenytoin/fosphenytoin (Figure 2).8,49,56,57 Patients with SE who receive these anesthetic agents will require intubation and mechanical ventilation, with hemodynamic monitoring in an ICU. Continuous electroencephalographic monitoring to document electrographic cessation of

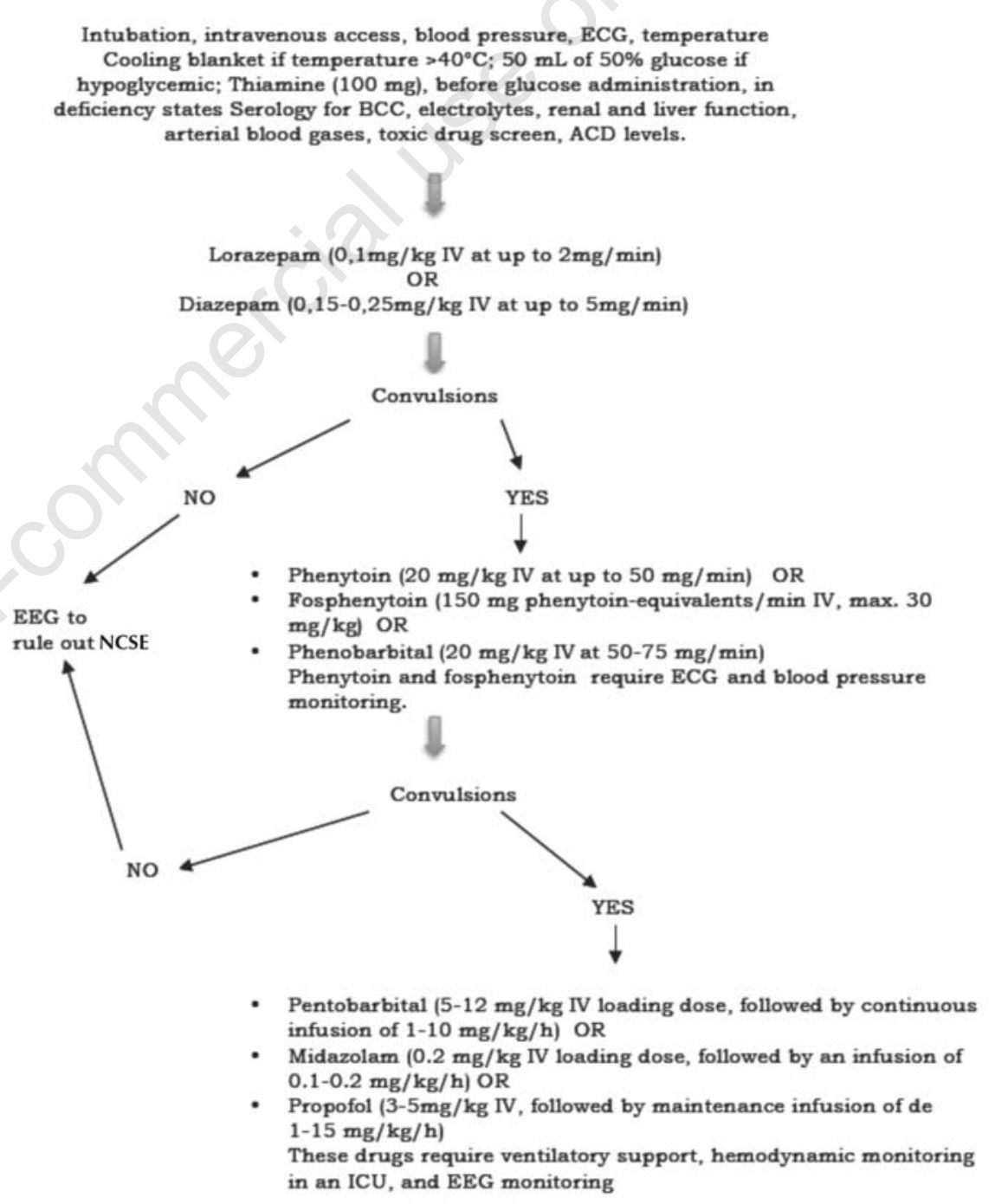

Figure 2. Chart flow for acute treatment of status epilepticus. ECG, electrocardiography; BCC, blood cell count; ACD, anticonvulsant drug; EEG, electroencephalogram; IV,intravenous; ICU, intensive care unit. 
seizures will also be needed. Hypotension caused by the depressed cardiac contractility and depressed vascular tonus is a very common adverse effect.3,5,8,49

\section{Pentobarbital}

Pentobarbital is probably the best studied intravenous drug used for RSE, besides being more accessible and cheaper than propofol or midazolam.5,58 It has an onset of action of 15$20 \mathrm{~min}$ and a half-life of $20-60 \mathrm{~h}$. It is preferable to phenobarbital because of its shorter elimination half-life, allowing a shorter duration of sedation and a more timely assessment of the patient's baseline clinical mental status. Pentobarbital is initially administered at an intravenous loading dose of $3-12 \mathrm{mg} / \mathrm{kg}$, over one hour, followed by continuous infusion of 1 $10 \mathrm{mg} / \mathrm{kg} / \mathrm{h}$, titrated to the desired EEG effect. ${ }^{5,6}$ While the patient is in a pentobarbital coma, EEG is the only reliable method to assess cessation of convulsive seizures, and the EEG pattern should change from epileptiform patterns to either burst-suppression or electrocerebral inactivity.3,6,8 Although according to various studies pentobarbital is efficacious in the treatment of RSE, it has been associated with high mortality.5,6,8,58 Infusion should be slowed down if profound hypotension occurs, with addition of pressor agents if needed. 8

\section{Midazolam}

Midazolam is a water-soluble benzodiazepine with a short half-life of 4-6 hours and rapid onset of action. It is effective and safe for RSE treatment.5,59 Because of its short elimination half-life, midazolam causes less prolongated sedation and less hypotension than the barbiturates. The disadvantages of midazolam infusion include its high cost and tachyphylaxis requiring substantial dose escalation after 1-2 days to maintain seizure control.8,46,49 With prolonged infusion, the drug accumulates as a result of an increase in elimination half-life, potentially leading to increased difficulty in bringing patients back to alertness. ${ }^{8}$ Use of the highest doses of midazolam, administered gradually, eventually demands ventilatory and circulatory support.46 The initial loading dose is $0.2 \mathrm{mg} / \mathrm{kg}$ as a slow intravenous bolus, followed by an infusion of $0.1-2.0 \mathrm{mg} / \mathrm{kg} / \mathrm{h}$, titrated to suppression of electrographic seizures. ${ }^{46,49}$

\section{Propofol}

Propofol is an intravenous anesthetic agent that has a short half-life (30-60 min) and, like midazolam, has been used for the induction and maintenance of anesthesia and also sedation in the ICU. It is highly lipophilic and has a large volume of distribution; it is, therefore, rapidly taken up by the brain and in turn rapid- ly eliminated. In contrast to midazolam, there is no accumulation with prolonged infusion, 8,46 promoting rapid recovery of consciousness and less interference in hemodynamic parameters. ${ }^{46} \mathrm{~A}$ loading dose of 1 to $2 \mathrm{mg} / \mathrm{kg}$ is administered over 5-10 min, followed by infusion of 1 to $15 \mathrm{mg} / \mathrm{kg}$, titrated to EEG seizure suppression. ${ }^{60}$ Because of its rapid clearance, propofol can induce withdrawal seizures and, therefore, it is recommended that it be tapered slowly at a rate of $5 \%$ of the maintenance infusion per hour.6,60 One small retrospective study of 14 patients compared propofol and midazolam infusion for RSE, and demonstrated both were equally effective in clinical and electrographic seizure control, although midazolam had fewer side effects (Figure 2). ${ }^{61}$

\section{Topiramate}

Topiramate, an ACD with multiple action mechanisms can be effective in terminating RSE.46,62 Dosages range from 300 to 1600 $\mathrm{mg} /$ day given in three doses daily and administered in suspension form via nasogastric tube, titrated up to 24-72 h.6,46,62 Published case series make reference only to lethargy as an important adverse effect; however, more information is needed before definite conclusions can be made about its role in RSE. Furthermore, the lack of an intravenous form makes acute administration of topiramate difficult. ${ }^{8}$

\section{Conclusions}

SE is a common neurological event in the elderly. It has a high mortality rate, particularly because it is usually related to cerebrovascular disease, systemic metabolic diseases and others comorbidities. As many elderly patients may not show typical signs of convulsive status, there is a great risk of seizure maintenance for an excessive period of time, thus increasing the likelihood of permanent neurological damage. On the other hand, NCSE is a group of highly heterogeneous clinical conditions ranging from mild impairment of consciousness to deep coma, requiring an EEG for diagnosis. Furthermore, in the elderly pharmacokinetics are complex and, in the context of a serious illness and polypharmaceutical use, aggressive treatment of NCSE may also become a risk. However, rapid intervention with prompt and aggressive onset of GCSE treatment shortens the duration of the status and also lowers its case fatality rate.

Further research into the etiology, diagnosis and treatment of SE in this rapidly growing elderly population are needed in order to provide better care and to avoid neuronal damage or even death.

\section{References}

1. Rona S, Rosenow F, Arnold S, et al. A semiological classification of status epilepticus. Epileptic Disord 2005;7:5-12.

2. DeToledo JC. Changing presentation of seizures with aging: clinical and etiological factors. Gerontology 1999;45:329-35.

3. Willmore LJ. Epilepsy emergencies: the first seizure and status epilepticus. Neurology 1998;51:S34-8.

4. Waterhouse E, Towne A. Seizures in the elderly: nuances in presentation and treatment. Cleve Clin J Med 2005;72Suppl 3:S26-37.

5. Waterhouse EJ, DeLorenzo RJ. Status epilepticus in older patients: epidemiology and treatment options. Drugs Aging 2001;18:133-42.

6. Towne AR. Epidemiology and outcomes of status epilepticus in the elderly. Int Rev Neurobiol 2007;81:111-27.

7. ILAE. Proposal for revised clinical and electroencephalographic classification of epileptic seizures. From the Commission on Classification and Terminology of the International League Against Epilepsy. Epilepsia 1981;22:489-501.

8. Kandula P, Harden C. Management of status epilepticus. In: Schapira AHV. Neurology and clinical neuroscience. Philadelphia: Mosby Inc; 2007. pp. 719-31.

9. Lowenstein DH, Bleck T, Macdonald RL. It's time to revise the definition of status epilepticus. Epilepsia 1999;40:120-2.

10. Blume WT, Luders HO, Mizrahi E, et al. Glossary of descriptive terminology for ictal semiology: report of the ILAE task force on classification and terminology. Epilepsia 2001;42:1212-8.

11. Lowenstein DH. The management of refractory status epilepticus: an update. Epilepsia 2006;47Suppl1:35-40.

12. Varelas PN, Mirski MA. Status epilepticus. Curr Neurol Neurosci Rep 2009;9:469-76.

13. Kaplan PW. Assessing the outcomes in patients with nonconvulsive status epilepticus: nonconvulsive status epilepticus is underdiagnosed, potentially overtreated, and confounded by comorbidity. J Clin Neurophysiol 1999;16:341-53.

14. Hesdorffer DC, Logroscino G, Cascino G, et al. Incidence of status epilepticus in Rochester, Minnesota, 1965-1984. Neurology 1998;50:735-41.

15. DeLorenzo RJ, Hauser WA, Towne AR, et al. A prospective, population-based epidemiologic study of status epilepticus in Richmond, Virginia. Neurology 1996;46:1029-35.

16. Wu YW, Shek DW, Garcia PA, et al. Incidence and mortality of generalized 
convulsive status epilepticus in California. Neurology 2002;58:1070-6.

17. Vignatelli L, Tonon C, D’Alessandro R. Incidence and short-term prognosis of status epilepticus in adults in Bologna, Italy. Epilepsia 2003;44:964-8.

18. Knake S, Rosenow F, Vescovi M, et al. Incidence of status epilepticus in adults in Germany: a prospective, population-based study. Epilepsia 2001;42:714-8.

19. Coeytaux A, Jallon P, Galobardes B, Morabia A. Incidence of status epilepticus in French-speaking Switzerland: (EPISTAR). Neurology 2000;55:693-7.

20. Tejeiro J, Gomez Sereno B. [Status epilepticus]. Rev Neurol 2003;36:661-79. [Article in Spanish].

21. DeLorenzo R. Clinical and epidemiology study of status epilepticus in the elderly. Newton, MA: Butterworth-Heinemann; 1997.

22. Krumholz A. Epidemiology and evidence for morbidity of nonconvulsive status epilepticus. J Clin Neurophysiol 1999;16: 314-22.

23. Shneker BF, Fountain NB. Assessment of acute morbidity and mortality in nonconvulsive status epilepticus. Neurology 2003;61:1066-73.

24. Korn-Lubetzki I, Steiner-Birmanns B, Galperin I, et al. Nonconvulsive status epilepticus in older people: a diagnostic challenge and a treatable condition. J Am Geriatr Soc 2007;55:1475-6.

25. Forcadas I. [Status epilepticus in the elderly]. Neurologia 1997;12 Suppl 6:46-53. [Article in Spanish].

26. Kaplan PW. Nonconvulsive status epilepticus. Neurology 2003;61:1035-6.

27. Sheth RD, Drazkowski JF, Sirven JI, et al. Protracted ictal confusion in elderly patients. Arch Neurol 2006;63:529-32.

28. Rosenow F, Hamer HM, Knake S. The epidemiology of convulsive and nonconvulsive status epilepticus. Epilepsia 2007;48Suppl8:82-4.

29. Sung CY, Chu NS. Status epilepticus in the elderly: etiology, seizure type and outcome. Acta Neurol Scand 1989;80:51-6.

30. DeLorenzo RJ, Pellock JM, Towne AR, Boggs JG. Epidemiology of status epilepticus. J Clin Neurophysiol 1995;12:316-25.

31. Rumbach L, Sablot D, Berger E, et al. Status epilepticus in stroke: report on a hospital-based stroke cohort. Neurology 2000;54:350-4.

32. Tatum WOt, Ross J, Cole AJ. Epileptic pseudodementia. Neurology 1998;50:14725.

33. Velioglu SK, Ozmenoglu M, Boz C, Alioglu Z. Status epilepticus after stroke. Stroke 2001;32:1169-72.

34. De Reuck J, Van Maele G. Status epilepticus in stroke patients. Eur Neurol 2009;62: 171-5.

35. DeLorenzo RJ, Towne AR, Pellock JM, Ko D. Status epilepticus in children, adults, and the elderly. Epilepsia 1992;33 Suppl4:S15-25.

36. Towne AR, Pellock JM, Ko D, DeLorenzo RJ. Determinants of mortality in status epilepticus. Epilepsia 1994;35:27-34.

37. Waterhouse EJ, Vaughan JK, Barnes TY, et al. Synergistic effect of status epilepticus and ischemic brain injury on mortality. Epilepsy Res 1998;29:175-83.

38. Litt B, Wityk RJ, Hertz SH, et al. Nonconvulsive status epilepticus in the critically ill elderly. Epilepsia 1998;39: 1194-202.

39. Van Cott AC. Epilepsy and EEG in the elderly. Epilepsia 2002;43Suppl3:94-102.

40. Rowan AJ, Ramsay RE, Collins JF, et al. New onset geriatric epilepsy: a randomized study of gabapentin, lamotrigine, and carbamazepine. Neurology 2005;64:186873.

41. Kellinghaus C, Loddenkemper T, Dinner DS, et al. Seizure semiology in the elderly: a video analysis. Epilepsia 2004;45:263-7.

42. Lowenstein DH, Aminoff MJ. Clinical and EEG features of status epilepticus in comatose patients. Neurology 1992;42:100-4.

43. Lee SI. Nonconvulsive status epilepticus. Ictal confusion in later life. Arch Neurol 1985;42:778-81.

44. Towne AR, Waterhouse EJ, Boggs JG, et al. Prevalence of nonconvulsive status epilepticus in comatose patients. Neurology 2000;54:340-5.

45. Leppik IE, Derivan AT, Homan RW, et al. Double-blind study of lorazepam and diazepam in status epilepticus. JAMA 1983;249:1452-4.

46. Garzon E. Estado de Mal Epiléptico. J Epilepsy Clin Neurophysiol 2008;14:7-11.

47. Fischer JH, Patel TV, Fischer PA. Fosphenytoin: clinical pharmacokinetics and comparative advantages in the acute treatment of seizures. Clin Pharmacokinet 2003;42:33-58.

48. Treiman DM, Meyers PD, Walton NY, et al. A comparison of four treatments for generalized convulsive status epilepticus.
Veterans Affairs Status Epilepticus Cooperative Study Group. N Engl J Med 1998;339:792-8.

49. Bleck TP. Management approaches to prolonged seizures and status epilepticus. Epilepsia 1999;40Suppl1:S59-63.

50. Pluhar J, Birnbaum, AK, Graves, NM. Valproic acid serum concentrations and daily dose in elderly nursing home residents: effects of age, gender, and concomitant medications. Epilepsia 1999;40:70.

51. Beyenburg S, Bauer J, Reuber M. New drugs for the treatment of epilepsy: a practical approach. Postgrad Med J 2004;80: 581-7.

52. Rossetti A0, Bromfield EB. Levetiracetam in the treatment of status epilepticus in adults: a study of 13 episodes. Eur Neurol 2005;54:34-8.

53. Beyenburg S, Reuber M, Maraite N. Intravenous levetiracetam for epileptic seizure emergencies in older people. Gerontology 2009;55:27-31.

54. Atefy R, Tettenborn B. Nonconvulsive status epilepticus on treatment with levetiracetam. Epilepsy Behav 2005;6:613-6.

55. Lowenstein DH, Alldredge BK. Status epilepticus at an urban public hospital in the 1980s. Neurology 1993;43:483-8.

56. Treiman DM, Walton, NY, Collins NF, Point, P. Treatment of status epilepticus if first drug fails. Epilepsia 1999;40:243.

57. Lowenstein DH, Aminoff MJ, Simon RP. Barbiturate anesthesia in the treatment of status epilepticus: clinical experience with 14 patients. Neurology 1988;38:395-400.

58. Yaffe K, Lowenstein DH. Prognostic factors of pentobarbital therapy for refractory generalized status epilepticus. Neurology 1993;43:895-900.

59. Parent JM, Lowenstein DH. Treatment of refractory generalized status epilepticus with continuous infusion of midazolam. Neurology 1994;44:1837-40.

60. Stecker MM, Kramer TH, Raps EC, et al. Treatment of refractory status epilepticus with propofol: clinical and pharmacokinetic findings. Epilepsia 1998;39:18-26.

61. Prasad A, Worrall BB, Bertram EH, Bleck TP. Propofol and midazolam in the treatment of refractory status epilepticus. Epilepsia 2001;42:380-6.

62. Towne AR, Garnett LK, Waterhouse EJ, et al. The use of topiramate in refractory status epilepticus. Neurology 2003;60:332-4. 